\section{Intersections}

Canadian Journal of Music

Revue canadienne de musique
Intersections CANADIAN JOURAL OF MUSIC
REVUE CANADIENEE DE MUSIOUH

\title{
Les nouveaux enjeux de la granulation sonore : l'esthétique populaire de l'Intelligent Dance Music (IDM)
}

\section{Anthony Papavassiliou}

Volume 30, numéro 2, 2010

URI : https://id.erudit.org/iderudit/1006380ar

DOI : https://doi.org/10.7202/1006380ar

Aller au sommaire du numéro

\section{Éditeur(s)}

Canadian University Music Society / Société de musique des universités canadiennes

\section{ISSN}

1911-0146 (imprimé)

1918-512X (numérique)

Découvrir la revue

Citer cet article

Papavassiliou, A. (2010). Les nouveaux enjeux de la granulation sonore : l'esthétique populaire de l'Intelligent Dance Music (IDM). Intersections, 30(2), 101-116. https://doi.org/10.7202/1006380ar
Résumé de l'article

L’Intelligent Dance Music, abrégé IDM, est un courant né dans les années 1990 en Angleterre. Sa particularité a été de proposer une musique aux spécificités structurelles populaires (rythmes majoritairement binaires, nombreuses répétitions, formes classiques, etc.) mêlée à une certaine complexité du contenu rythmique et timbral. Reprenant les techniques de micromanipulations sonores explorées dans les milieux académiques des années 1970 et 1980, les artistes d'IDM les ont développées par la création d'outils dédiés qu'ils ont ensuite diffusés à un large public appartenant à une nouvelle génération d'auditeurs " actifs ». Ce partage, favorisé par le contexte particulier de développement des technologies numériques qui débuta dans les années 1990, a donné lieu à une utilisation massive des micromanipulations dans les musiques populaires. L'esthétique issue des micromanipulations se manifeste à l'audition par l'effet de granulation du son qui provient de l'utilisation de diverses techniques de construction et décomposition tels que la granulation sonore, la synthèse granulaire, le découpage, répétition et mélange automatisés, ainsi que l'étirement ou encore le « gel » du son. Le présent article résume l'évolution de l'usage des micromanipulations sonores en tenant compte du contexte qui a favorisé son développement et présente l'IDM comme le courant qui a assuré le transfert d'un concept de création vers une esthétique populaire.
All Rights Reserved (C) Canadian University Music Society / Société de musique des universités canadiennes, 2011
Ce document est protégé par la loi sur le droit d'auteur. L'utilisation des services d'Érudit (y compris la reproduction) est assujettie à sa politique d'utilisation que vous pouvez consulter en ligne.

https://apropos.erudit.org/fr/usagers/politique-dutilisation/ 


\title{
LES NOUVEAUX ENJEUX DE LA GRANULATION SONORE : L'ESTHÉTIQUE POPULAIRE DE L'INTELLIGENT DANCE MUSIC (IDM)
}

\author{
Anthony Papavassiliou
}

\section{INTRODUCTION}

En 2010, l'album These Hopeful Machines de Brian Transeau (BT) est nominé aux Grammy Awards dans la catégorie «Best Electronic/Dance Album». Outre la production irréprochable et l'énergie typique des œuvres de musiques électroniques populaires qui caractérisent l'ensemble des œuvres de cet album, l'esthétique générale présente des particularités propres au style développé par l'Intelligent Dance Music (IDM), un courant musical à la fois populaire et expérimental qui a émergé dans les années 1990 : synthétiseurs et samples se désagrégeant et se reconstituant en synchronisation avec la battue, sons inversés, tronqués, étirés, "gelés», et autres effets temporels de rupture, de transition ou d'agrément...

Si cet album est désormais important, c'est parce qu'il est le premier d'IDM à recevoir une telle attention des experts de l'industrie du disque. Par ses caractéristiques hybrides, il représente ce que l'on pourrait considérer comme le début d'une phase de maturité de la musique électronique, l'aboutissement d'un long processus de développement symbolisé par l'acquisition progressive d'une maîtrise des possibilités offertes par les nouvelles technologies. L'objectif de cet article sera de montrer qu'il existe, depuis les années 2000 dans les musiques électroniques populaires actuelles, une esthétique particulière héritée de l'Intelligent Dance Music. Cette esthétique, née d'un désir d'exploiter les possibilités d'explorations sonores offertes par les technologies électroniques et numériques, se manifeste par l'utilisation rythmique et dynamique d'éléments sonores réduits dans le temps, et doit son existence à ce que nous appellerons ici les micromanipulations sonores.

Dans une première partie, nous décrirons le concept visé par l'utilisation du terme «micromanipulation", puis nous aborderons le contexte historique de son apparition. Dans un second temps, nous présenterons l'IDM en mettant l'emphase sur l'esthétique que dégagent les micromanipulations opérées par les artistes qui constituent ce courant musical.

\section{DÉFINITION DE LA MICROMANIPULATION SONORE}

Face à la nécessité d'utiliser un terme qui puisse décrire le type de manipulations sonores rendues accessibles par les technologies numériques, nous nous 
sommes permis d'en emprunter un au vocabulaire scientifique, plus précisément à celui de la biologie. En effet, nous avions besoin, pour le présent travail, de décrire une pratique de création et d'édition musicales non pas basée sur le concept traditionnel de la note, mais participant à la constitution d'une matière sonore par l'utilisation d'éléments dont la durée peut varier entre l'échelle micro (plus de 1000 événements par seconde) et l'échelle de la matière elle-même.

Le terme "micromanipulation» est employé depuis les années 1970 pour définir une "opération effectuée sur un objet microscopique, dans le champ du microscope (microdissection, injections dans les cellules, microchirurgie, etc.) à l'aide d'instruments très petits (micro-instruments) et de dispositifs spéciaux (micromanipulateurs) ${ }^{1} »$. Dans la plupart des cas, l'ordinateur fait office de dispositif spécial tandis que les logiciels de conception sonore font office d'instruments permettant à l'homme d'agir à très petite échelle. Une micromanipulation sonore, que nous définirons comme étant une opération effectuée sur des micro-éléments sonores à l'aide d'outils permettant la création ou l'édition du son à très petite échelle, permet l'élaboration d'un matériau musical où le rythme d'apparition des événements traverse les différentes échelles pour former un ensemble à la fois complexe et cohérent. Dans les chapitres suivants, nous verrons que les artistes d'Intelligent Dance Music créent une petite variété d'effets rythmiques caractéristiques des micromanipulations sonores. L'esthétique du "micro-élément» sonore, qui va émerger et se répandre dans les musiques électroniques plus populaires, trouve son origine dans l'élaboration de la granulation sonore et de la synthèse granulaire.

\section{CONTEXTE HISTORIQUE}

L'esthétique qui découle des micromanipulations du son doit son existence à un contexte historique particulier, mêlant découvertes scientifiques, ingénierie pratique, bouleversements sociaux et remises en questions artistiques. En effet, ces cinquante dernières années ont donné lieu à une communication étroite et à des échanges complexes entre les différentes disciplines qui ont collaboré pour faire de la musique ce qu'elle est devenue aujourd'hui. Contrairement aux siècles précédents, la frontière séparant l'artiste de l'ingénieur ou l'auditeur de l'artiste tend à s'estomper : les compositeurs créent leurs instruments et diffusent leurs techniques de création tandis que des amateurs ou des professionnels développent des outils facilitant la mise en pratique de ces techniques.

\section{Ère pré-numérique}

Si l'histoire du développement des technologies qui ont conduit à une esthétique des micromanipulations sonores a été continue, on peut distinguer des phases successives où certaines disciplines ont dominé ou engendré l'activité des autres. Avant le début du XXe siècle, l'idée d'une maîtrise du son à l'échelle micro était purement théorique. On peut trouver des traces de cette volonté

\footnotetext{
1 «Micromanipulation» dans le Grand Robert de la langue française, consulté le o8 juin 2011.
} 
prématurée dans les écrits de Francis Bacon (The New Atlantis, 1626) lorsqu'il décrit un studio de production sonore utopique d'où émergent des harmonies faites de «fractions de sons». Le Gambara de Balzac (1837) émet également l'idée que la musique doit être envisagée comme la constitution d'une multitude d'éléments réduits, faisant ainsi l'apologie de la science pour son rôle fondamental vis-à-vis du développement de l’art : «Ce qui étend la science étend l'art. ${ }^{2} »$.

On peut sans aucun doute attribuer au physicien et inventeur Dennis Gabor la première application d'une pensée qu'il a lui-même développée plus tôt : en s'inspirant des récentes découvertes en physique quantique, ses recherches dans le domaine acoustique vont l'amener à construire dans les années 1940 un «granulateur de son" nommé Kinematical Frequency Convertor (Roads 2001). Le principe de la granulation sonore, qui permet pour la première fois de changer la hauteur d'un son indépendamment de sa durée, est le suivant : pour pouvoir augmenter la durée d'un son, il suffit de dupliquer certaines parties, tandis que pour raccourcir le son il suffit d'en enlever. Pour ne pas détruire la séquence sonore initiale, l'enjeu est de faire en sorte que la partie sélectionnée soit la plus petite possible. En théorie donc, la meilleure qualité que l'on pourrait obtenir serait un traitement dont la séquence, ou plutôt le grain compte tenu de la taille (ou durée) de l'élément concerné, serait infiniment petit. Cette application, entièrement mécanique 3 , inspira Pierre Schaeffer et Jacques Poulin qui construisirent le Phonogène. Ces derniers proposèrent deux premières versions en 1953 qui n'étaient que des appareils permettant de faire varier en relation la hauteur et la durée d'une bande magnétique 4 , puis une dernière version vit le jour en 1963. Nommée Phonogène Universel, elle permit enfin de traiter indépendamment hauteur et durée.

La granulation, qui fut ensuite développée pour permettre une riche variété de manipulations sonores et de méthodes de compositions, est une technique majeure permettant une élaboration affranchie du concept traditionnel de la note. La première œuvre qui explora cette technique serait Concret $\mathrm{PH}$, une œuvre électroacoustique de Iannis Xenakis réalisée en 1958 dans les studios du Groupe de Recherches Musicales (GRM). Avec cette nouveauté, le compositeur n'atteindra pas des durées de grains exceptionnellement faibles (les plus petits fragments feraient une seconde), mais sa manière de les arranger fera de lui l'un des précurseurs des musiques à base de granulation sonore. En effet, de nombreuses musiques électroacoustiques composées au GRM étaient constituées d'éléments véritablement courts (les Études de Boulez et de Stockhausen en 1952 ou encore Timbres-Durées de Messiaen en 1953), mais il s'agissait jusqu'alors d'arrangements particulièrement sommaires qui n'avaient rien à voir avec une démarche visant à constituer un ensemble sonore homogène à partir d'éléments fondamentaux. Comme le précise Xenakis :

2 Balzac, p.27.

3 Le Kinematical Frequency Convertor était une machine qui fonctionnait mécaniquement, cependant Gabor avait également envisagé une version électrique qu'il ne mit jamais au point.

4 Reproduisant l'effet obtenu lors du ralentissement ou de l'accélération d'un disque vinyle. 
Start with a sound made up of many particles, then see how you can make it change imperceptibly, growing and developing, until an entirely new sound results.... This was in defiance of the usual manner of working with concrète sounds. Most of the musique concrète which had been produced up to the time of Concret PH is full of many abrupt changes and juxtaposed sections without transitions. This happened because the original recorded sounds used by the composers consisted of a block of one kind of sound, then a block of another, and did not extend beyond this. I seek extremely rich sounds (many high overtones) that have a long duration, yet with much internal change and variety. Also, I explore the realm of extremely faint sounds highly amplified. There is usually no electronic alteration of the original sound, since an operation such as filtering diminishes the richness. (Xenakis 1972, Notes de programme du LP «Iannis Xenakis : Electro-Acoustic Music», New York : Nonesuch Records H-71246)

Après Concret PH, c'est Analogique B (1959) de Xenakis qui intègre encore une nouveauté : au lieu d'utiliser des "grains» d'éléments sonores déjà existants, il se servira d'enregistrements de sons purs produits par un générateur analogique. Ce n'est pas encore ce que l'on nommera plus tard de la synthèse granulaire, puisque les grains, bien qu'il s'agisse de sons synthétisés, sont toujours des éléments préenregistrés 5 . Cependant, les concepts du grain sonore se dessinent et l'utilisation des techniques stochastiques (qui ne sont pas sans rappeler les lois de la physique quantique) cède peu à peu la place aux ensembles sonores complexes, résultats d'un assemblage de «sons élémentaires adéquatement disposés dans le temps» (Xenakis 1992, p. 43).

La synthèse granulaire, granulation produite à partir d'éléments de synthèse, viendra des studios de Cologne (WDR), réputés pour avoir été le berceau de la synthèse sonore. La première œuvre, réalisée en 1960 à l'aide d'un générateur d'impulsions analogique nommé «multivibrateur ${ }^{6}$ ", sera Kontakte de Stockhausen. Cette œuvre avant-gardiste offre ici un ensemble de microélaborations d'une qualité qui ne sera égalée que de nombreuses années plus tard. C'est le cas notamment du passage à 5 '3o où un son granulé navigue entre différentes hauteurs au gré de la densité des grains qui le composent pour se détacher sur un Fa4 percutant, puis lentement se désagréger en un flux discontinu de grains devenus notes.

\section{Ère numérique}

Si les techniques d'édition et de génération analogiques ont permis de repousser les limites de la création musicale en offrant, entre autres, la possibilité d'agir sur le son de plus en plus précisément, ce n'est qu'avec les technologies numériques que les compositeurs ont pu enfin acquérir une liberté totale de manipulation.

5 Un des défauts majeurs d'Analogique B relevé par Roads (2001), sans doute dû à la limitation des techniques d'échantillonnage de l'époque, est l'absence de variation de durée des grains sonores.

6 Circuit électronique permettant de générer des impulsions à intervalles variables, permettant la granulation sonore ainsi que la synthèse granulaire entièrement contrôlées de manière électronique. 
L'avantage de la technologie numérique (ou digitale) sur la technologie analogique est qu'elle autorise, par l'échantillonnage ${ }^{7}$, la segmentation de n'importe quel signal en un ensemble de parties microscopiques. Par exemple, un son échantillonné 44100 fois par seconde, comme dans le cas du disque compact $(\mathrm{CD})$, donnera un ensemble « découpé» dont chaque élément aura pour durée environ 0 , $000 \mathrm{O} 2$ secondes, soit 20 microsecondes.

Il a cependant fallu que les ordinateurs deviennent assez performants pour pouvoir traiter de telles données. Jusqu'à la fin des années 1950, les ordinateurs appelés mainframes étaient d'immenses machines ne pouvant produire le moindre son :

The mainframes were gigantic, often taking up rooms or even entire floors. Early models had no monitors or screens, programs had to be submitted via punch cards, and the results delivered as printouts. Computing resources were severely constrained. It was difficult even to gain access to a mainframe - they were not commodity items and were centralized and available mostly at academic and research institutions (in 1957 the hourly cost to access a mainframe was $\$ 200$ !). [...] Programs were carefully designed and tuned to yield the highest efficiency.

The early algorithmic composition experiments were conducted with mainframes, and only sought to produce a symbolic score (just as a list of characters rather than any notation), certainly without synthesized realization. (Ge Wang 2007, p.57).

En 1957, Max Mathews qui vient de mettre au point l'un des tous premiers convertisseurs numérique-analogique adapté aux mainframes, développe MUSIC, un langage de programmation permettant de concevoir de la musique sur un ordinateur. Dès lors, les équipements informatiques de l'époque ont pu émettre les sons programmés par l'artiste-technicien. Avec une telle utilisation de l'ordinateur comme interprète exclusif, le compositeur devient obligatoirement le facteur des instruments qu'il utilise. Bien sûr, ce dernier pourrait tout à fait enregistrer sa composition et s'en servir comme matériel dans l'élaboration d'une œuvre mixte, mais l'enjeu qui se profile est bien plus grand : il s'agit d'avoir à sa disposition un «instrument total» pouvant produire n'importe quel son ou musique, et dont les seules limites seraient l'imagination et la maîtrise de l'artiste. Pourtant, une limitation technologique existe encore à cette époque. D’une part, on note que les ordinateurs n'étaient pas encore assez puissants pour effectuer des calculs compliqués (on ne pouvait, par exemple, pas encore effectuer de manipulations en temps réel). D’autre part, parce que MUSIC était un environnement de développement simple, il fallait donc développer des algorithmes évolués de conception sonore pour faciliter et enrichir le panel des possibilités créatrices du compositeur.

Les années 1970 et 1980 furent à la fois la période de développement le plus important des technologies informatiques, et la période d'élaboration

7 Plus de précisions sur l'échantillonnage ou encore les différents termes utilisés ici, tels que «numérique», «analogique» ou «digital» dans Curtis Roads 2007, p. 3-41. 
d'algorithmes de production sonore de plus en plus riches et puissants (le second n'ayant sans doute pas pu être possible sans le premier). Les compositeurs engagés dans cette aventure avaient à réactualiser sans cesse les outils qu'ils développaient à des fins artistiques. Le cas de Curtis Roads illustre bien ce phénomène : en 1974, il développe à l'aide de MUSIC V ${ }^{8}$ le premier logiciel de synthèse granulaire sur un Burroughs B670o de l'Université de Californie à San Diego. Pourtant, la technologie du mainframe de cette époque ne permettait pas encore de faire varier la durée des grains, fixés à $40 \mathrm{~ms}$. Il réalise alors Klang-1 qu'il considère comme une expérimentation technique (Roads, 2001). Un an plus tard, il compose Protoype, une étude dérivée de Klang-1 qui intègre des durées de grains plus courts (20 ms), ainsi que des variations d'enveloppe. Puis en 1986, Barry Truax compose Riverrun, une pièce pour laquelle il introduit la synthèse granulaire en temps réel. Une autre étape est alors franchie : la densité des grains varie de 100 à 2000 éléments par seconde, chacun d'entre eux ayant une fréquence et une durée propres.

Les années 1980 furent également le début d'une accessibilité croissante des technologies numériques au grand public avec, en 1983, l'apparition de la norme MIDI et la sortie du premier synthétiseur digital à succès populaire ${ }^{9}$, le Yamaha DX-7. Durant cette décennie, les micro-ordinateurs firent leur apparition sur le marché, les algorithmes s'améliorèrent, les premiers séquenceurs graphiques (Pro 16 ${ }^{10}, 1984$ ) et logiciel interactifs (Music Mouse, 1985) virent le jour et la sortie de Max, un nouvel environnement de programmation sonore graphique, laissait présager le meilleur pour l'élaboration musicale à venir.

Le récent développement des technologies de création et d'édition sonore a permis à une nouvelle génération de compositeurs de voir le jour. Il est désormais possible de produire tout type de musique électronique à moindre frais. C'est ce contexte favorable à une rencontre entre la recherche expérimentale et la pratique populaire qui donne naissance à l'Intelligent Dance Music.

\section{L'Intelligent Dance Music}

L'IDM est un courant musical né au début des années 1990 qui a pour origine la parution d'une compilation sous le label WARP Records nommée «Artificial Intelligence». Cet album réunissant des artistes novateurs et peu connus de la scène électronique tels qu'Aphex Twin (Richard D. James) ou encore le duo Autechre (Rob Brown et Sean Booth), fut reçu par la critique et les amateurs comme un manifeste ${ }^{11}$. Kurt B. Reighley, dans l'article Downtempo de l'ouvrage «Modulations : Une histoire des musiques électroniques», raconte ainsi sa

8 Un «descendant» de MUSIC.

9 Notamment grâce à son coût relativement réduit; cinquante fois moins cher que celui des appareils de la génération précédente (Mathews, 2006).

10 Logiciel qui deviendra le désormais célèbre Cubase en 1989.

11 L'abréviation "IDM" pour Intelligent Dance Music provient en effet du nom donné à une liste de discussion publique ouverte sur Internet par Alan Parry le 1er août 1993. Cette liste était en grande partie dédiée aux débats concernant les œuvres et les artistes présents sur la compilation Artificial Intelligence, comme le rapporte Adam Winfield dans son article «Is IDM Dead? » paru dans Igloo Magazine le 24 novembre 2007 (http://igloomag.com/features/is-idm-dead, consulté le 8 juin 2011). 
rencontre avec l'IDM lorsqu'il écrivait des articles sur les dernières parutions dans le monde des musiques électroniques :

Au milieu des années 9o, la pop a failli me faire expulser de chez moi. Non, je ne mettais pas mes disques trop forts pour les oreilles délicates de mes voisins... En fait, j'avais perdu tout désir de seulement écouter un disque. Je me sentais tellement découragé par ce médium qui se mordait la queue et ne circulait plus qu'en cercles restreints que mes écrits en étaient devenus tristes et mornes. [...].

Je mentirais en disant que la séminale compilation Artificial Intelligence, sortie sur le label Warp de Sheffield, a immédiatement charmé mes perspicaces oreilles de critique. La vérité est vraiment ailleurs. Certains de mes collègues parlaient entre eux de gens comme Richard James (Aphex Twin, Polygon Window, The Dice Man), Autechre ou Black Dog, qui, tous, apparaissaient sur le disque Artificial Intelligence... J'ai mené mon enquête, tout simplement. Warp avait compris que la vitalité de la techno et de la house diminuait rapidement en raison du nombre de plus en plus grand de morceaux interchangeables qui se contentaient de reproduire des formules codifiées [...]. Warp promettait plus qu'une fugace montée d'adrénaline, et mettait en avant une équipe d'artistes engagés dans une logique de carrière et dont les ouvres complexes et subtiles ne se contentaient pas d'un effet de satisfaction immédiate. (Reighley 2004.)

L'IDM prend racine dans un contexte particulier qui est l'aboutissement de trente années d'expérimentations techniques. La particularité de ce mouvement est qu'il a été initié par des artistes non issus des milieux académiques précédemment décrits, mais sensibles à cette philosophie d'exploration et de dépassement de la tradition musicale qui se perpétuait dans les œuvres électroniques populaires de l'époque ${ }^{12}$. Les artistes comme Aphex Twin ou Autechre étaient avant tout des mélomanes habitués et amateurs de la production musicale populaire qui expérimentaient avec des appareils abordables tels que des synthétiseurs «grand public» et des ordinateurs personnels. Cependant, plutôt que de se limiter aux outils mis à leur disposition pour pratiquer leur art, ils décidèrent de développer les leurs dans la continuité de leurs prédécesseurs électroacousticiens.

L'IDM, qui n'en est qu'à ses débuts, propose d'emmener la musique électronique populaire de l'époque hors des délimitations convenues de la dance, de la techno, de la house ou de leurs dérivés naissants. La recette est relativement simple : conserver l'accessibilité musicale par une structure et des formules résolument populaires (mesures en 4/4, synthétiseurs standards, kits de batteries standards, ...) tout en proposant une certaine complexité rythmique et

12 Les succès populaires parmi lesquels figurent des œuvres telles que Go de Moby (1991) ou encore I Like to Move It d'Erick Morillo (1994) étaient presque entièrement constitués de sons électroniques, pourtant ces sons étaient moins utilisés pour leur originalité que pour leur "présence». Le timbre des différents instruments était très facilement reconnaissable et reproductible à l'aide des synthétiseurs courants et les différents éléments musicaux étaient organisés en flux mélodiques simples, ce qui n'offrait pas de réelle nouveauté par rapport à la conception traditionnelle de l'instrumentation musicale. 
des effets tonals et timbraux à la fois inattendus et pertinents. Plus que des simples trouvailles et effets compositionnels, on y trouve également une volonté de rupture par la transgression, phénomène que l'on connaît bien dans le développement du langage tonal ou celui du rock. Cette «rébellion» musicale s'exprime de manière fugace dans Polygon Window lorsque la mélodie s'engouffre dans une série de fausses notes (3'o1") avant de se reprendre puis s'éteindre, ou de manière plus franche dans Preminition où le timbre de la basse, désaccordée et résonnante, est en opposition totale avec ce que l'on attend habituellement d'une basse à cette époque.

L'esthétique de la micromanipulation sonore à l'œuvre dans l'IDM ne se fixe réellement que quelques années après la parution d'Artificial Intelligence, dans le milieu des années 1990, avec la sortie des premiers albums produits séparément par les artistes phares du courant que sont Autechre, Aphex Twin, Boards of Canada ou encore Amon Tobin (Ninja Tune).

Alors que dans les années 1970 et 1980 l'idée d'une exploration sonore dépassant le concept d'unité indivisible de la note s'exprimait principalement dans le développement de techniques granulaires, les artistes d'IDM utilisèrent un ensemble de techniques de découpage et de réutilisation des sons pour agrémenter de différentes façons le discours de leurs œuvres. Cette démarche, qui visait à créer une esthétique musicale plutôt qu'une exploration à la fois technique et contemplative, avait pour but de transformer les stratégies compositionnelles en fonctions narratives au sein d'œuvres aux éléments variés : lorsqu'on écoute Point Line Cloud (Curtis Roads, 2005), nous avons affaire à l'expression totale et unique d'une technique de micromanipulation sonore; le flux musical semble dénué de structure et donne la sensation de ne jamais vraiment aboutir.

Dans 1.618 (BT, 2006), œuvre typique d'IDM, c'est le contraire qui se produit : même si nous sommes constamment confrontés à une esthétique "granulaire» du son, il est souvent difficile de dire quelle technique a été utilisée pour la produire. Selon la fonction désirée, l'effet est différent et pourtant, nous distinguons une évidente unité esthétique, celle d'un matériel musical semblant être constitué d'un ensemble d'atomes sonores.

\section{NAISSANCE DE L'ESTHÉTIQUE BASÉE SUR LES MICROMANIPULATIONS SONORES}

Lalbum Richard D. James ${ }^{13}$ (Aphex Twin, 1996) est probablement le premier ensemble d'œuvres qui ont fixé le style de l'IDM. Contrairement aux tâtonnements d'Artificial Intelligence, on y retrouve la manifestation d'un usage quasi-systématique des micromanipulations sonores. 4 (1996) de Richard D. James, est un exemple simple du mélange entre complexité rythmique et simplicité structurelle que vont entre autres proposer les artistes d'IDM. On retrouve une forme rondo composée de mélodies pentatoniques accompagnées par des violons, le tout supporté par deux patterns rythmiques alternés : un premier dont le roulement de la caisse claire oscille entre une cadence élevée et une cadence

13 Comme cela peut porter à confusion, il vaut mieux préciser que le nom de l'album est aussi le véritable nom d'Aphex Twin qui est le pseudonyme le plus connu de Richard D. James. 
proche du son continu, un second où la caisse claire se contente de frapper les deuxième et quatrième temps de chaque mesure. Le schéma ci-dessous résume les premières mesures de manière relativement simplifiée.

Les rectangles de la couche "Caisse claire» représentent les patterns d'accélération et de décélération de la caisse claire, technique de micromanipulation du son qui n'est plus noyée dans un océan intemporel de sons divers comme c'était le cas trente ans auparavant, mais utilisée comme réel élément du discours musical. L'effet est ici geste : l'accélération du rythme semble viser le même type d'affect que ceux provoqués par une descente de notes jouée au piano ou encore un glissando vers le bas d'un violon. La décélération joue le même rôle mais dans le sens contraire. Cette œuvre qui fait l'ouverture du premier album d'Aphex Twin après quatre années de préparation présente d'une part, aux auditeurs de musiques électroniques populaires une œuvre élaborée, d'autre part, aux oreilles plus exigeantes une réelle structure musicale donnant un sens à l'utilisation des micromanipulations.

C'est d'ailleurs dans un souci de compréhension et d'assimilation que Richard D. James prendra toujours soin de correctement structurer ses œuvres, de procéder avec justesse aux répétitions / variations et d'opposer dans de nombreux cas la richesse du rythme à la simplicité harmonique. Notons d'ailleurs que la grande majorité des œuvres d'IDM utilisent le pentatonisme pour leur matériel mélodique et harmonique.

Dans Peek 824545201, Aphex Twin superpose les éléments composés de grains sonores et fait varier leur densité indépendamment tout en conservant leur synchronisation avec la métrique de l'œuvre (voir figure 1).

Les micromanipulations sont effectuées de façon très méticuleuse dans Yellow Calx ou encore To Cure a Weakling Child, dont les éléments vocaux ne sont pas sans rappeler Gesang der Jünglinge de Stockhausen. Cette fois encore, la répétition y est manifestement présente, mais d'une manière moins "didactique » que dans 4 puisqu'il ne s'agit plus d'une alternance de patterns rythmiques

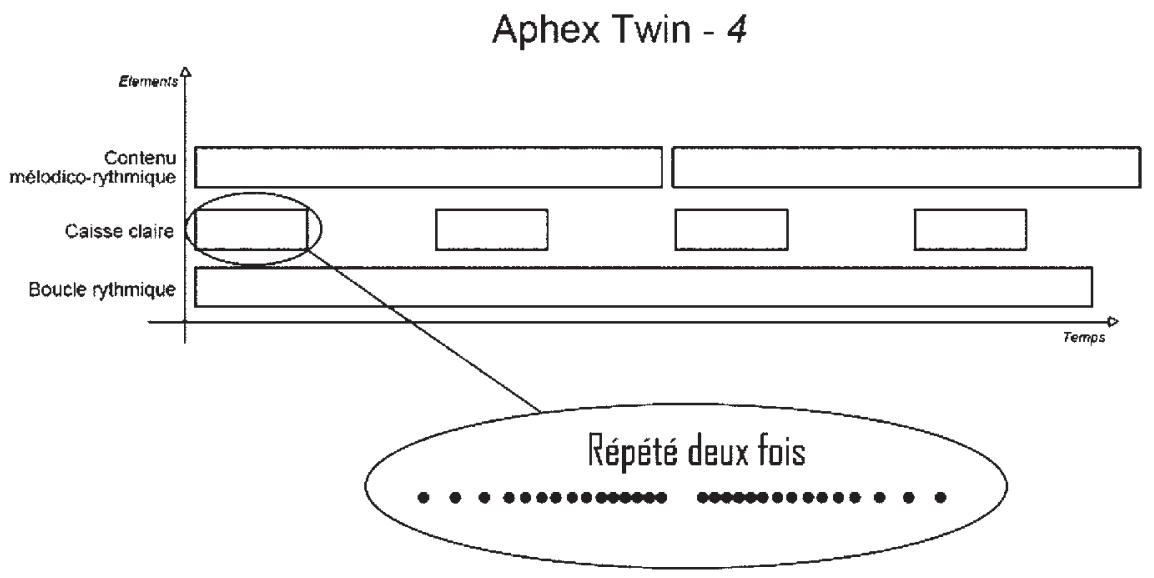

Figure 1. 

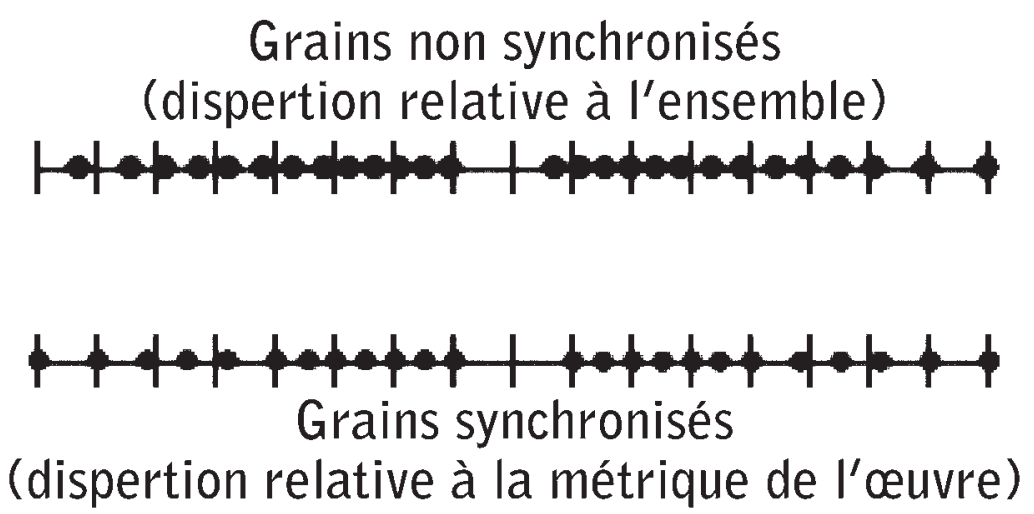

Figure 2. Différences entre des grains dont les variations de fréquence (ou densité) évoluent selon des règles propres à l'ensemble de grains et des grains dont les variations de fréquence évoluent de sorte à correspondre aux règles métriques qui régissent l'ensemble de la composition. Une synchronisation relatant le rapport « ensemble de grains / ensemble de l'œuvre » qu'il ne faudra pas confondre avec le rapport " synchrone / asynchrone " qui sert à qualifier le type de flux des grains propre à la granulation sonore et à la synthèse granulaire et donc la qualité du timbre obtenue à forte densité (hauteur définie / nuage).

mais plutôt de variations au sein d'une progression (ajout progressif d'éléments) de l'ensemble de l'œuvre.

La particularité dans ces œuvres est la mise en commun de techniques d'enrichissement rythmiques classiques que sont les effets de résonance (reverb, delay) et de découpage simple (ce que BT appellera plus tard Stutter Edit ${ }^{14}$ ), avec les différents emplois de la granulation. L’ensemble de ces stratégies compositionnelles desservent le même objectif : créer une esthétique du quantum sonore, d'une matière qui se compose et se décompose pour former des flux rythmiques conditionnant certaines perceptions mélodiques et timbrales.

Dans Tewe (1997) d'Autechre, tous les éléments de la composition sont composés de grains dont la densité va fluctuer en permanence au gré de l'évolution de l'ensemble. On observe alors une micro-polyrythmie, qui deviendra une spécialité du duo anglais. Non seulement ce traitement de la granulation propose-t-il une richesse inouïe de combinaisons sonores tout en gardant la musicalité de l'ensemble, mais permet-il également de confondre subtilement mélodie et rythme ou encore d'opérer à des variations d'une métrique dominée par les rapports complexes qu'entretiennent les éléments de son contenu, comme c'est le cas dans VI Scose Poise (Autechre, 2001).

14 Brian Transeau s'attribue à tort la paternité de cette technologie, sans doute pour mieux promouvoir le logiciel du même nom qu'il vend depuis peu. Le concept est assez simple : c'est un outil «dérivé» de ceux permettant la granulation, dans le sens où il est principalement dédié à la découpe et la répétition d'éléments sonores en rythme avec la battue générale de l'œuvre. Il fait partie de ces nombreux logiciels qui ont été développés pour simplifier et automatiser les micromanipulations «simples» qui étaient autrefois faites manuellement (voir le chapitre suivant : Le «feedback loop» d'Aphex Twin). 


\section{LE « FEEDBACK LOOP» D'APHEX TWIN}

Dans une interview de 2006, Aphex Twin déclarait : «The funniest feedback loop is when people make plug-ins that try to emulate what I do and then I end up using them - thanks ${ }^{15} »$. Cette citation résume bien la situation qui a contribué à développer et diffuser l'esthétique engendrée par l'utilisation quasi systématique des micromanipulations sonores. À l'origine, les artistes d'IDM concevaient leur propres algorithmes ou logiciels d'élaboration sonore pour produire à leur guise et de manière efficace (productive) les effets qu'ils voulaient obtenir. Au moment où l'IDM a émergé, la plupart des foyers commencèrent à s'équiper d'ordinateurs personnels et de plus en plus d'auditeurs se mirent à composer leur propre musique. Très vite, un marché est né : les séquenceurs virtuels se dotèrent de la capacité d'être équipés de plug-ins ${ }^{16}$ tiers (Cubase VST, 1996), des environnements de développement d'applications audio et de plug-ins virent le jour (Max/MSP, 1997, SynthEdit, 1999, Synthmaker, 2006) et les premiers manglers ${ }^{17}$ apparurent. C'est par exemple le cas du désormais célèbre Glitch ${ }^{18}$ (Kieran Foster, 2005), un plug-in gratuit permettant d'accomplir un certain nombre de micromanipulations devenues par la suite standards. C'est ainsi que Native Instruments, un éditeur de logiciels audio, s'est associé avec l'artiste d'IDM Tim Exile pour intégrer à son catalogue les logiciels (The Finger, 2009 et The Mouth, 2010) que ce dernier avait développé quelques années auparavant pour ses compositions. Collaboration étonnante par ailleurs si l'on tient compte du fait que Tim Exile est l'un des rares artistes d'IDM à avoir généreusement partagé son savoir et ses outils ${ }^{19}$ avec la communauté. Ce dernier continue néanmoins à participer activement à la diffusion de l'esthétique des micromanipulations en donnant régulièrement des conférences et diverses présentations en ligne sur sa technique et ses outils. Plus récemment (2010), c'est l'éditeur de logiciels de traitements sonores Izotope qui s'est associé avec Brian Transeau pour commercialiser un logiciel que ce dernier développe pour son utilisation personnelle depuis plus de dix ans. Nommé Stutter Edit, l'outil offre des possibilités similaires à celles proposées par les nombreux manglers disponibles sur le marché.

15 B. Murnin 2007.

16 Un plug-in est un petit logiciel prévu pour être utilisé au sein d'un autre logiciel, dit hôte.

17 Un Sound Mangler (littéralement Destructeur de Son), est le nom donné à cette génération de plug-ins conçus pour reproduire les effets utilisés dans l'IDM ou encore le courant Glitch en offrant des options de «destruction» en tout genre tels que l'inversion, la répétition, la "dé-quantification", le mélange, le «gel» et une multitude d'autres effets originaux applicables en temps réel.

18 Le nom est emprunté au courant musical minimal qui suivit un développement parallèle à celui de l'IDM. Le courant glitch est essentiellement focalisé sur l'utilisation de sons courts dont le timbre fait principalement référence aux sons non désirés par les productions électroniques modernes tels que les craquements et autres erreurs numériques (K. Cascone 2004, p. 392-398).

19 En 2008 Tim Exile partage au public un «mangler» qu'il a développé pour sa propre utilisation. La démo est alors téléchargeable gratuitement avec la note suivante (la page ayant été supprimée, une seule source témoigne encore de la note originale : http://www.djtechtools.com/forum/ showthread.php?t=524, consulté le 8 juin 2011) : "This is the super-cut-down demo of one of the features of the Reaktor patch which I programmed and use live». L'outil permet notamment d'effectuer toutes les opérations en temps réel depuis le clavier d'ordinateur, facilitant d'autant plus l'accès à la technologie. 


\section{AnATOMIE DE $1.618^{20}$ (This Binary Universe, Brian Transeau 2006)}

1.618 est un bon exemple d'un usage raffiné des micromanipulations sonores dix ans après la sortie de l'album Richard D. James d'Aphex Twin. L'œuvre exploite avec une certaine délicatesse l'ensemble des techniques élaborées pendant ces années-là. Une nappe fait l'ouverture du morceau, supportée par un ensemble de grains dont la hauteur et le rythme varient au cours du temps donnant l'effet d'un liquide en ébullition. La nappe, qui pourrait tout à fait être un accord de synthétiseur, rappelle l'effet provoqué par le Paul's Extreme Sound Stretch ${ }^{21}$ développé en 2006 et qui permet d'étirer n'importe quel son jusqu'à $1^{\star} 10^{18}$ fois sa durée initiale. L'algorithme est si efficace que l'effet obtenu est une sorte de " gel» de la matière dont la subtile évolution confère à la séquence traitée l'aspect d'une texture à la fois organique et éthérée. Des cordes pincées sont ensuite introduites puis inversées, décomposées et répétées pour aboutir sur un ensemble "granuleux» simplement structuré : chaque pulsation des mesures en 4/4 reproduit une hauteur définie. Si la qualité de la production est indéniablement meilleure que celle des premiers albums d'IDM, la recette reste la même. La structure rythmique est simple, une simili caisse claire (ici un son "aspiré» en alternance aiguë/ grave) marque les deuxième et quatrième temps, tandis que le contenu musical est composé d'une multitude d'effets rythmiques voyageant entre l'échelle microscopique et celle de la note. On note que pour cette composition, BT n'abandonne pas l'usage classique de la mélodie, comme c'est parfois le cas des œuvres d'IDM les plus radicales, révélant une démarche identique à celle du choix de la structure et qui confère au style un côté séduisant et accessible.

\section{UNE ESTHÉTIQUe DEVENUE POPUlaIRE}

Si l'IDM perdure de nos jours en tant que courant autonome avec des artistes tels que BT, Seefeel, Proem, Dimlite ou Raoul Sinier, la reprise des différentes techniques de micromanipulations sonores par des artistes de tous bords a eu pour effet, d'une part, la création de nouveaux genres de musiques électroniques, et, d'autre part, l'enrichissement de genres déjà existants. La création de nouveaux genres musicaux sur la base d'une reprise de certains aspects de l'IDM a non seulement contribué à populariser les micromanipulations mais également à élargir, tout en semant une certaine confusion dans les dénominations, le champ d'expression des effets rythmiques et timbraux mis au point

201.618 représente le nombre d’or. Ce genre de références aux nombres et aux mathématiques est très présent dans l'IDM (Boards of Canada dissémine nombre d'entre elles dans ses œuvres, pour le plus grand bonheur de ses fans), ce qui est très certainement le résultat des facultés que les artistes d'IDM ont dû développer pour composer les outils servant à produire leurs œuvres.

21 L'auteur, qui distribue son logiciel gratuitement sur Sourceforge.net, précise dans les notes : "This program doesn't process the sound as a single piece: it cuts the sound in small pieces and process them. Each small piece is called a "window". The size of the windows controls the size of the window in samples, which affects the frequency and the time resolution of the resulting sounds.» (http:// hypermammut.sourceforge.net/paulstretch/, consulté le 8 juin 2011). 
par les pionniers de l'Intelligent Dance Music. Le breakcore22, par exemple, est un genre constitué de musiques particulièrement rapides (généralement audessus de $160 \mathrm{bpm}$ ) composées avec une majorité d'éléments très courts. Très proche de l'IDM, elle est née de la volonté de produire une musique "hard$\operatorname{cor}^{23}$ » complexe. Il existe également des genres qui n'ont pas encore trouvé de dénomination et qui ont pourtant permis à l'IDM de se renouveler en prenant de nouvelles formes. C'est le cas du hip-hop expérimental de Luke Vibert ou bien de l'IDM-folk psychédélique de Bibio utilisée par ailleurs dans de nombreuses publicités ${ }^{24}$ (L.L. Bean, Toyota, Amazon, ...).

L'enrichissement de genres populaires par les techniques de composition de l'IDM s'est quant à lui effectué de manière beaucoup plus prudente. Cela semble naturel quand on sait que ce qui garantit la popularité d'une œuvre tient pour beaucoup de l'utilisation d'un certain nombre de codes permettant sa "compréhension" par un maximum d'auditeurs. Intégrer à ses œuvres des éléments d'une esthétique nouvelle est un risque que peu d'auteurs à succès sont prêts à prendre. Bjork fait partie des premiers artistes pop' à avoir intégré l'utilisation de micromanipulations sonores dans ses œuvres avec l'album Vespertine (2001) qui sort pendant la période d'affirmation des artistes de Warp Records; les rythmes d'Aurora (Bjork 2001) rappellent fortement l'esthétique de l'album Chiastic Slide (Autechre) sorti quatre années plus tôt. Autechre a également été une influence pour le célèbre groupe Radiohead qui sort pendant la même période deux albums rappelant les expérimentations de l'IDM : Kid A (2000) et Amnesiac (2001). Toujours durant la même période, certains artistes de house music, courant de musiques électroniques populaires composées en grande partie de samples, commencent à réduire la durée des leurs échantillons sonores. Ainsi est né le «micro-sampling» pour désigner ces éléments sonores destinés au contenu mélodico-harmonique dont la durée n'excède que rarement le quart de seconde, comme c'est le cas dans New-Jack (2007) du groupe Justice.

Ces artistes qui se mirent à modifier leur pratique pour y intégrer des éléments de plus en plus réduits ont été les premiers à tirer parti des méthodes de compositions explorées quelques années plus tôt par les artistes de l'Intelligent Dance Music. À l'écoute des œuvres de Bjork, Justice ou encore Bibio, on se rend compte que malgré la disparité de style, il existe un point commun qui est l'expression d'une esthétique minutieuse et précise. Cette esthétique résulte

${ }_{22}$ Mt saint michel + saint michaels mount (Aphex Twin 2001) est un exemple parfait de breakcore. Le compositeur canadien Aaron Funk est probablement l'artiste le plus prolifique et le plus talentueux du courant.

23 Le mot hardcore est souvent utilisé en préfixe pour désigner la variante «rapide» d'un genre. Il est parfois remplacé par le suffixe "core», comme dans le cas du mot breakcore qui est très certainement la contraction de "hardcore breakbeat».

24 Si les mélodies folk de Bibio rendent ses œuvres accessibles à un large public et offrent de ce fait un matériau sonore exploitable par des publicitaires, il est intéressant de noter un phénomène d'utilisation des micro-éléments sonores dans les jingles d'émissions en tous genres : pour être au goût du jour, nombreuses sont les émissions à avoir intégré dans leurs génériques des éléments de rythmes rapides ou des fractions de sons répétés. 
d'une manipulation sonore opérée à une échelle pratiquement inexplorée avant le développement des technologies électroniques et numériques.

\section{CONCLUSION}

La technologie numérique ayant ouvert les portes à une infinité d'élaborations sonores, il était nécessaire de dépasser notre façon d'envisager la musique et les formes que nous lui avions associées. Il a alors fallu que des compositeurs ingénieux assurent la transition d'une conception harmonique héritée de la période classique vers une organisation de la matière libérée du poids de la tradition instrumentale. La granulation sonore et la synthèse granulaire avaient offert une première alternative aux démarches entreprises par les compositeurs souhaitant explorer plus avant les possibilités du timbre. L'idée qui consistait à redéfinir l'échelle du plus petit constituant musical permettait non seulement la possibilité d'une manifestation organique du son, mais également d'étendre l'action du rythme au-delà des frontières de l'expressivité du corps.

Les artistes de l'Intelligent Dance Music ont joué un rôle majeur dans ce processus d'exploration en développant le concept de la granulation et en lui adjoignant l'utilisation de différentes techniques d'altération, de découpage et de répétition des composants sonores. En proposant l'ensemble de ces techniques que nous avons librement nommées «micromanipulations sonores » et en les intégrant au sein d'œuvres plus ou moins "conventionnelles», l'IDM crée une esthétique qui s'exprime sur deux plans. D'une part, celle-ci se manifeste par une complexification et une densification du rythme, par des effets d'altération temporelle des différents éléments musicaux, ainsi que par des effets de constitution et de dégradation de la matière sonore. D’autre part, l'esthétique de l'IDM exprime, par l'application des techniques de découpage et de dégradation du son, la rupture d'avec les attentes de l'écoute traditionnelle en se servant de la temporalité de l'œuvre et de ses constituants comme support à un schéma de tension/détente alternatif. Cela n'exclut évidemment pas, dans un monde futur hypothétique où l'esthétique de l'IDM serait devenue la norme, la persistance d'un ensemble de lois héritées de la pratique instrumentale; la situation actuelle tend simplement à prouver, à l'image des découvertes scientifiques du début du $\mathrm{XX}^{\mathrm{e}}$ siècle, qu'une conception «quantique» du son peut cohabiter avec une conception classique de l'œuvre musicale. Mieux, cela montre qu'il peut exister une contamination des musiques populaires par des pratiques «savantes » grâce au partage et à la diffusion massive des outils et de la connaissance.

\section{RÉFÉRENCES}

Balzac, Honoré de. 2010. Gambara. http://www.ebooksgratuits.com/pdf/balzac _gambara.pdf. [consulté le 10 mai 2011].

Cascone, Kim. 2004. "The Aesthetics of Failure : "Post-Digital" Tendencies in Contemporary Computer Music». Audio culture : readings in modern music, sous la dir. de Cristoph Cox et Daniel Warner. New York : Continuum. 
Collins, Karen. 2008. From Pac-Man to Pop Music, Interactive Audio in Games and New Media. Ashgate.

Wang, Ge. 2007. "A history of programming and music». The Cambridge Companion to Electronic Music, sous la dir. de Nick Collins et Julio d'Escrivàn. Cambridge : Cambridge University Press.

Cuen, Leticia. 2007. «Le geste sonore et le geste cinétique : une évocation des sensations et des émotions humaines». Temps, Geste et Musicalité, sous la dir. de Michel Imberty et Maya Gratier. Paris : L'Harmattan.

Demers, Joanna. 2010. Listening through the noise. Oxford : Oxford University Press.

De Poli, Giovanni, Aldo Piccialli, et Curtis Roads. 1991. Representations of Musical Signals. Cambridge : MIT Press.

Gabor, Denis. 1946. «Acoustical quanta and the theory of hearing». Nature 159 (4044) : 591-594.

Miranda, Eduardo Reck. 2002. Computer Sound Design, Synthesis Techniques and Programming. Oxford: Elsevier.

Murnin, Brian. 2006. «Aphex Twin Interview». http://www.clashmusic.com/ feature/aphex-twin. [consulté le 1o mai 2011].

Roads, Curtis. 2001. Microsound. Cambridge : MIT Press.

2007. L'audionumérique, musique et informatique. Paris : Dunod.

Schaeffer, Pierre. 2002. De la musique concrète à la musique même. Montréal : Mémoire du Livre.

Shapiro, Peter et Caipirinha Productions. 2004. Modulations : une histoire de la musique électronique. Paris : Editions Allia.

Stockhausen, Karlheinz. 1962. "The concept of unity in electronic music». Perspectives of New Music 1, $\mathrm{n}^{0} 1: 39$.

Truax, Barry. 1990. «Composing with Real-Time Granular Sound». Perspectives of New Music Vol. 28, $\mathrm{n}^{\circ} 2$ : 120-134.

Young, Rob. 2005. Warp : labels unlimited. London : Black Dog Publishing.

Xenakis, Iannis. 1992. Formalized music. New York : Pendragon Press.

\section{RÉSUMÉ}

L'Intelligent Dance Music, abrégé IDM, est un courant né dans les années 1990 en Angleterre. Sa particularité a été de proposer une musique aux spécificités structurelles populaires (rythmes majoritairement binaires, nombreuses répétitions, formes classiques, etc.) mêlée à une certaine complexité du contenu rythmique et timbral. Reprenant les techniques de micromanipulations sonores explorées dans les milieux académiques des années 1970 et 1980, les artistes d'IDM les ont développées par la création d'outils dédiés qu'ils ont ensuite diffusés à un large public appartenant à une nouvelle génération d'auditeurs «actifs». Ce partage, favorisé par le contexte particulier de développement des technologies numériques qui débuta dans les années 1990, a donné lieu à une utilisation massive des micromanipulations dans les musiques populaires. L'esthétique issue des micromanipulations se manifeste à l'audition par l'effet de granulation du son qui provient de l'utilisation de diverses techniques de construction et décomposition tels que la granulation sonore, la synthèse granulaire, le découpage, 
répétition et mélange automatisés, ainsi que l'étirement ou encore le «gel» du son. Le présent article résume l'évolution de l'usage des micromanipulations sonores en tenant compte du contexte qui a favorisé son développement et présente l'IDM comme le courant qui a assuré le transfert d'un concept de création vers une esthétique populaire.

\begin{abstract}
Intelligent Dance Music (IDM) is a trend that emerged in England in the 199os. The music of the movement is characterized by popular structural elements (primarily binary rhythms, numerous repetitions, classical forms, etc.) combined with a certain rhythmic and timbral complexity. IDM artists use specific tools to recreate sonic micromanipulation techniques that were developed in academic circles in the rofos and 1980 s, which they distribute to a large public audience, who make up the next generation of 'active' listeners. Sharing these techniques has been facilitated through the development of digital technology that emerged at the beginning of the 1990s, which made it possible for micromanipulations to be widely used in popular music. Micromanipulations create an aesthetic that incorporates sound granulation and which is characterized by the diverse technologies used in the construction and decomposition techniques, such as granulation of sound, granular synthesis, cutting, repetition and automated mixes as well silence or pauses. This article summarizes the development of auditory micromanipulation techniques and the conditions that fostered its development, and introduces IDM as the trend which has introduced this technique into the popular aesthetic.
\end{abstract}

